\title{
THE SCHUR MULTIPLIERS OF THE MATHIEU GROUPS ${ }^{1)}$
}

\author{
N. BURGOYNE ${ }^{2)}$ and P. FONG
}

To the memory of TAdasi Nakayama

The Mathieu groups are the finite simple groups $M_{11}, M_{12}, M_{22}, M_{23}, M_{24}$ given originally as permutation groups on respectively 11, 12, 22, 23, 24 symbols. Their definition can best be found in the work of Witt [1]. Using a concept from Lie group theory we can describe the Schur multiplier of a group as the center of a "simply-connected" covering of that group. A precise definition will be given later. We also mention that the Schur multiplier of a group is the second cohomology group of that group acting trivially on the complex roots of unity. The purpose of this paper is to determine the Schur multipliers of the five Mathieu groups. We conclude:

the multipliers of $M_{11}, M_{12}, M_{23}, M_{24}$ are trivial;

the multiplier of $M_{22}$ is a cyclic group of order 3 .

The proof of this result occupies part II of this paper. The techniques used there differ somewhat from those introduced by I. Schur in his three impressive memoirs [2]. We hope that our methods may have an independent interest. ${ }^{*}$

In part I we collect certain elementary (and undoubtedly well-known) results on the Mathieu groups. Several of these results are necessary for the proofs in part II.

Both authors are greatly indebted to C. Sommerfield for his generous advice concerning many of the calculations connected with this work. We also kindly thank L. Evens and W. Wong for helpful correspondence on various matters.

PART I

Frobenius, in $\S 5$ and $\S 6$ of his work [3] on multiply-transitive groups,

Received September 3, 1965.

1) This work was partially supported by the Office of Naval Research (Contract Nonr 3656-09) and by the National Science Foundation (Contract NSF GP-1610).

2) Alfred P. Sloan Foundation Fellow.

*) Added in proof. These results have also been obtained by J. G. Thompson. 
determines the character tables of $M_{12}$ and $M_{21}$. He further states that he has calculated the characters of all known multiply-transitive groups of degree 24 or less. Unfortunately, he does not give these tables for $M_{11}, M_{22}$, and $M_{23}$. They are of course readily derived and we take the liberty of reproducing them here (see Tables 1, 2, 3). In referring to specific characters or conjugacy classes we shall always adopt the notation of reference [3].

In the following three sections we discuss 1) subgroups, 2) multiplytransitive permutation representations, and 3) automorphism groups of the Mathieu groups.

\section{§1. Certain Subgroups}

We restrict ourselves solely to those subgroups which will appear later in the paper. All the results of this section are either explicitly contained in [1] or [3], or else follow therefrom by straightforward considerations.

If $M_{11}$ is represented on eleven letters those subgroups fixing a letter are conjugate. We denote any one of these by $M_{10}$. Its Sylow 2-subgroup, and therefore the Sylow 2-subgroup of $M_{11}$, is semi-dihedral of order $16^{3)} M_{11}$ also contains the subgroup $\operatorname{PSL}(2,11)$.

$M_{24}$ contains $M_{12}$ as a subgroup. The intersection of $M_{12}$ with $M_{23}$ is $M_{11}$. We note that the index of $M_{12}$ in $M_{24}$, and $M_{11}$ in $M_{23}$, is in both cases relatively prime to 3 .

There also exists in $M_{24}$ a subgroup of index 3.11.23. This subgroup is the holomorph of the elementary abelian group of order 16. Thus $M_{24}$ contains $G L(4,2) \simeq A_{8}$. The representation of $A_{8}$ on 24 letters breaks up into three sets of transitivity containing $1,8,15$ letters. Thus $A_{8}$ is in fact a subgroup of $M_{23}$. As a group on 15 letters, $A_{8}$ has subgroups $G_{(1344)}$ fixing one letter. $G_{(1344)}$ may be described as a subgroup of $G L(4,2)$ fixing one non-zero vector. $G_{(1344)}$ is contained in $M_{22}$.

We denote by $M_{21}$ any one of the subgroups of $M_{22}$ with index 22 and fixing a letter. $\quad M_{21}$ is the simple group $\operatorname{PSL}(3,4)$. For use in the next section we note that $M_{21}$ does possess subgroups of index 21 and 56, but that all other subgroups have index greater than $56 .^{4)}$

3) $a^{8}=b^{2}=1, b^{-1} a b=a^{3}$.

4) R. W. Hartley, Annals of Math. Vol 27 (1925), pp. 140-158. 


\section{§ 2. Multiply-Transitive Permutation Representations}

Apart from the well-known multiply-transitive permutation representations of the Mathieu groups, there exists also a representation of $M_{11}$ as a triplytransitive group on 12 letters. The subgroup fixing one letter is $\operatorname{PSL}(2,11)$, and corresponds to the character $1+11$ of $M_{11}$. There are no further multiplytransitive representations of the Mathieu groups. With two exceptions, this is readily verified by inspecting the character tables and applying standard tests ([4], pp. 155-157). The two less obvious cases are the character $1+55$ of $M_{22}$ and the character $1+7 \cdot 36$ of $M_{24}$. The latter case is disposed of by noting that if there were such a subgroup of index 253 in $M_{24}$, the restriction of the character $\overline{11.21}$ to this subgroup would by direct calculation remain irreducible. Since 231 does not divide the order of this subgroup, this is a contradiction.

The question of whether $M_{22}$ has a subgroup of index 56 requires a few words more. Assume $M_{22}$ contains such a subgroup; call it $H$. As a subgroup of $M_{22}, M_{21}$ when represented on 56 letters must be transitive, as one may verify by computing the character $1+55$ on $M_{21}$. Thus each $M_{21}$ intersects $H$ in a subgroup of order 360 . Therefore $H$, as a group on 22 letters, must in its turn be transitive. Since $M_{22}$ is triply-transitive, two distinct $M_{21}$ 's intersect in a subgroup of order 960 and so must intersect $H$ in distinct subgroups of order 360 . Thus, the 22 distinct subgroups of index 22 in $H$, fixing a letter, form a single conjugacy class of $H$. By simple arguments one can deduce that they are maximal. $H$ is therefore primitive on 22 letters. By a theorem of Wielandt ${ }^{5)} H$ is doubly-transitive. But 21 does not divide the order of $H$, and so no subgroup of index 56 exists in $M_{22}$. In particular $M_{11}$ is not a subgroup of $M_{22}$ (It is possible to show independently that $M_{11}$ is not a subgroup of $M_{22}$ from the presentation of $M_{22}$ given by Witt [1], and the fact that $M_{11}$ can be generated by any two elements of order 4 and 11 respectively).

\section{§ 3. Automorphism Groups}

From the character tables of the relevant subgroups one finds that with the exception of $M_{12}$, each Mathieu group $M_{n}$ possesses a unique conjugacy class of subgroups of index $n, n=11,22,23,24$. The automorphism groups

5) H. Wielandt, Math. Zeit. Vol 63 (1956), pp. 478-485. 
of these $M_{n}$ thus coincide with their normalizers in the symmetric group of degree $n$. The normalizers are easily found (see Miller [5]). The result is that $M_{11}, M_{23}, M_{24}$ are complete, while $\operatorname{Aut}\left(M_{22}\right) / M_{22} \simeq Z_{2}$. The outer automorphism of $M_{22}$ is the one induced by its normalizer in $M_{24}$.

Now $M_{12}$ has two conjugacy classes of $M_{11}$ subgroups, and an outer automorphism which interchanges them. Also $M_{12}$ equals its normalizer in the symmetric group of degree 12 , Miller [5]. Thus Aut $\left(M_{12}\right) / M_{12} \simeq Z_{2}$.

PART II

This part is divided into five sections. In 1) we list some required results on multipliers and coverings. In 2) we outline a simple argument on induced characters which in 3) is used to show that $M_{11}, M_{12}, M_{23}, M_{24}$ each has a trivial multiplier. ${ }^{6)}$ The more involved case of $M_{22}$ is reserved for 4). Finally in 5) an entirely independent argument, using results of Brauer, is given for the non-existence of the 2-part in the multiplier of $M_{23}$.

\section{$\S 1$ The Schur Multiplier}

We shall follow closely the terminology of Schur [2]. By a covering (ergänzte Gruppe) of $H$ we mean a group $G$ with central subgroup $A$ such that $G / A \simeq H$. If $[G, G]$ contains $A$ we will call $G$ a proper covering of $H$.

In studying the projective representations of a finite group $H$, Schur was led to the problem of constructing such proper coverings of $H$. He called a proper covering $K$ of largest possible order a representation group of $H$. The central subgroup $M$, such that $K / M \simeq H$ and $[K, K]$ contains $M$, is called the multiplier of $H$. It is a finite abelian group. In general, $H$ can have several non-isomorphic representation groups as well as isomorphic ones coming from distinct central extensions of $H$. Nevertheless, the subgroup $M$ is the same in every case.

In one of his principal results, Schur proved that all projective representations of $H$ (over the complex numbers) can be considered as ordinary representations of any representation group $K$ of $H$. Conversely, those ordinary representations of $K$, not the identity on $M$, give projective representations of $H$.

If $H$ is simple, it possesses a uniquely determined representation group $K$,

6) i.e. what Schur calls an "abgeschlossene Gruppe". 
and $K$ is its own representation group. Any proper covering of $H$ is then of the form $G \simeq K / N$ where $N$ is some subgroup of the multiplier of $H$. We shall often consider coverings $G$ with $A \simeq Z_{p}$ a cyclic group of prime order $p$. If $H$ coincides with its commutator subgroup and such a covering is not proper, then $G \simeq H \times Z_{p}$.

We will need the following results on coverings and multipliers. Let $H$ be any finite group, $M$ its multiplier, and $K$ a representation group of $H$.

(a) The only primes that may occur in the order of $M$ are those that occur to at least the second power in the order of $H$ (Schur [2], 1904, p. 48).

(b) Let $S$ be a subgroup of index $r$ in $H$. Let $M^{(r)}$ denote those elements of $M$ with order relatively prime to $r$. Then $M^{(r)}$ is a subgroup of the multiplier of $S$ (Schur [2], 1904, p. 49).

(c) Consider those irreducible characters $\%$ of $K$ which on restriction to $M$ give a fixed linear character of $M$. Let $N$ be the kernel of this linear character. If $A=M / N$, then $A$ is a cyclic group $Z_{n}$ of order $n$. The characters $\chi$ can be considered as " $n$-valued" projective characters of $H$. Put $G=K / N$; then $G$ is a proper covering of $H$, and $G / A \simeq H$. If $c$ is a conjugacy class in $H$ and its inverse image in $G$ gives $n$ distinct classes of $G$, we say that $c$ splits in $G$. In the contrary case we say $c$ does not split in $G$. If $\bar{g} \in G$ is a particular inverse image of $g \in c$, then $c$ splits if and only if $\bar{g}$ is not conjugate in $G$ to any $\bar{g} a, a \in A, a \neq 1$. In sections 3) and 4), we will often wish to know which classes of $H$ do or do not split. A useful test: If an element $g \in c$ has order relatively prime to $n$, then $c$ must split in $G$. If $\%$ is one of the above projective characters of $H$, it takes values differing by multiples of $n$-th roots of unity on a class which splits. It vanishes on all classes which do not split in $G$ (Schur [2], 1904, §4).

(d) Let $\chi$ be one of the characters defined in (c). The degree of $\%$ is a multiple of $n$, and is also a divisor of the order of $H$ (Schur [2], 1904, pp. 4448).

(e) The semi-dihedral group has a trivial multiplier. The multiplier of $\operatorname{PSL}(2,11)$ is $Z_{2}$ (Schur [2], 1907, pp. 108, 119).

From (a) we see that the multiplier of a Mathieu group can at moxt contain a 2-primary and a 3-primary part. 


\section{§ 2. The Inducing Argument}

One of our main techniques for calculating multipliers is to try to obtain information about possible coverings of $H$ by inducing up linear characters of known coverings of a subgroup $S$ of $H$. This section sketches the method.

Assume $G$ is a proper covering of $H$, with $G / A \simeq H$. This gives a covering $T$ of $S, T / A \simeq S$. Now if we know, independently, that any such covering of $S$ is not proper, then $T$ will have at least one linear character $\phi$ which is not identically 1 on $A$. We denote by $\phi^{*}$ the character of $G$ induced by $\phi$. Each irreducible component of $\phi^{*}$, by the Frobenius reciprocity theorem, will be a projective character of $H$. Thus $\phi^{*}$ will vanish on certain non-splitting classes. Using the orthogonality condition that $\Sigma\left|\phi^{*}\right|^{2}$ is a multiple of the order of $G$, we can then often determine precisely which combinations of classes cannot split in $G$.

A special case is $A \simeq Z_{p}$ where $p$ is a prime, and $T \simeq S \times Z_{p}$ with $\phi$ the identity on $S$ and non-trivial on $Z_{p}$. Let $\pi$ be the permutation character of $H$ on the cosets of $S$, and suppose $\pi$ contains $n(\pi)$ irreducible components. Then the projective character $\phi^{*}$ will be $\pi(c)$ times a $p$-th root of unity on a class $c$ which splits. Let $\left\{c_{j}\right\}$ be the classes of $H$ which do not split and denote by $h_{j}$ the order of the centralizer in $H$ of an element in $c_{j}$. Then using results of $(c)$ of the previous section and the above orthogonality condition, we must have

$$
\sum_{j} \pi\left(c_{j}\right)^{2} / h_{j}=n, \text { where } n \text { is an integer. }
$$

Furthermore, $n(\pi)-n$ is the number of irreducible components of $\phi^{*}$. The formula $(*)$ will be used repeatedly in the next section.

\section{$\S 3$. Multipliers of $M_{11}, M_{12}, M_{23}, M_{24}$}

Since the Sylow 2-subgroup of $M_{11}$ is semi-dihedral, by (b) and (e) of $\S 1$, the 2-primary part of its multiplier is trivial. We next investigate the 2-part of the multiplier of $M_{12}$.

Consider the situation discussed at the end of $\S 2$, with $H=M_{12}, S=M_{11}$, and $A=Z_{2}$. Referring to Table 4, where the relevant $\pi$ is given $(n(\pi)=2)$, we see by applying the test of (c) in $\S 2$ that the (1), (11), $(3)^{3}$, and $(5)^{2}$ classes must certainly split. Now the (8)(2) class has centralizer $Z_{8}$ and the 
covering of $Z_{8}$ can only be $Z_{16}$ or $Z_{8} \times Z_{2}$. Both centralize an inverse image of an (8)(2) element. Thus the (8)(2) class also splits. For the remaining classes $(2)^{4},(4)^{2}$, and $(6)(3)(2)$, the sum in $(*)$ is less than 1 . Thus these classes also split and $\phi^{*}$ contains two irreducible components. Restricting back to $M_{11} \times Z_{2}$, we see that these components must be of dimension 1 and 11 . By (d) of $\S 2$ the assumed covering of $M_{12}$ is not proper. Therefore the 2-part of the multiplier of $M_{12}$ is trivial.

The calculation for $M_{12}$ has been given in detail since it will be repeated, with only minor variations, in the next four cases.

For the 2-part of the multiplier of $M_{23}$ we assume that the 2-part of $M_{22}$ is trivial. This will be proved independently in the next section. Using the inducing argument with $S=M_{22}$, we see that only the $(2)^{8},(4)^{4}(2)^{2}$, and $(6)^{2}(3)^{2}(2)^{2}$ classes could not split. Again by $(*)$ they must split and etc. Thus the 2-part of the multiplier of $M_{23}$ is trivial.

For $M_{24}$ take $S=M_{23}$. The only classes (see Table 5 ) that could not split are the $(2)^{8},(4)^{4}(2)^{2},(8)^{2}(4)(2),(6)^{2}(3)^{2}(2)^{2}$ and the two $(14)(7)(2)$ classes. Again by $(*)$ they must split and etc. Thus the 2-part of the multiplier of $M_{24}$ is trivial.

For the 3-part of the multiplier of $M_{11}$, use $S=P S L(2,11), A=Z_{3}$, and (e) of $\S 2$. Only the $(3)^{3}$ and $(6)(3)(2)$ classes could not split. By $(*)$ they must and etc. Thus the 3-part of the multiplier of $M_{11}$ is trivial. To show the 3part of the multiplier of $M_{12}$ is trivial, use $S=M_{11}$, and the same arguments as before. The 3-parts of the multipliers of $M_{23}$ and $M_{24}$ are trivial by the above results for $M_{11}$ and $M_{12}$ and (b) of $\S 2$.

\section{$\S 4$. The Multiplier of $M_{22}$}

The 3-part of the multiplier of $M_{22}$ is easily determined with the help of a generalization of a theorem of Grün by Swan [6]. In our special case this theorem states that if $H$ is $p$-normal, the $p$-part of its multiplier is the same as that of the normalizer of the center of a Sylow $p$-subgroup. Now $M_{22}$ and $M_{21}$ have the same Sylow 3-subgroup $Z_{3} \times Z_{3}$. They also have the same normalizers (of order 72) of the Sylow 3-subgroup. The 3-part of the multiplier of $M_{21}$ is $Z_{3}$. This follows because $S L(3,4)$ is a proper covering and the multiplier of $Z_{3} \times Z_{3}$ is $Z_{3}$. Thus the 3-part of the multiplier of $M_{22}$ is also $Z_{3}$. 
We now prove that the 2-part of the multiplier of $M_{22}$ is trivial. Assume that $\bar{M}_{22} / Z_{2} \simeq M_{22}$ is a covering of $M_{22}$. The proof will consist of the following steps :

(i) Show that the covering of $G_{(1344)}$ in $\bar{M}_{22}$ is a direct product.

(ii) With $S=G_{(1344)}$ use the inducing argument to show that all classes of $M_{22}$ must split in $\bar{M}_{22}$.

(iii) Show that if all its classes split, the Sylow 2-subgroup of $M_{22}$ has no proper covering, and hence $\bar{M}_{22} \simeq M_{22} \times Z_{2}$.

Let $P$ denote the Sylow 2-subgroup of $M_{22}$. For our purpose, $P$ is best described as the Sylow 2 -subgroup of $S L(3,4)$ extended by the field automorphism $\left(x_{i j}\right) \rightarrow\left(x_{i j}^{2}\right)$, where $x_{i j} \in G F(4), i, j=1,2,3$. $P$ contains two normal, elementary abelian subgroups of order 16 ; call one of them $E$. Then $P / E \simeq$ $D_{8}$, the dihedral group of order 8 . This is a semi-direct product. Consider $E$ as a 4 -dimensional vector space over $G F(2)$; then $P$ can be given as an affine group $P=\{(B, \vec{b})\}$,

$$
\vec{x} \rightarrow B \vec{x}+\vec{b}, \quad \vec{x}, \vec{b} \in E
$$

and $\{B\} \simeq D_{8}$. One finds the following generators for $D_{8}$ :

$$
B_{1}=\left(\begin{array}{ll}
I & I \\
0 & I
\end{array}\right) \quad B_{2}=\left(\begin{array}{ll}
J & 0 \\
0 & J
\end{array}\right) \quad B_{3}=\left(\begin{array}{cc}
I & K \\
0 & I
\end{array}\right)
$$

where $I=\left(\begin{array}{ll}1 & 0 \\ 0 & 1\end{array}\right), \quad J=\left(\begin{array}{ll}1 & 1 \\ 0 & 1\end{array}\right), \quad K=\left(\begin{array}{ll}0 & 1 \\ 1 & 1\end{array}\right) . \quad$ A particular maximal subgroup of $P$ is $P_{1}$, the group generated by $E$ and $\left\{B_{1}, B_{2}\right\}$. By direct calculations one may verify that $P_{1}$ is isomorphic to a Sylow 2-subgroup of $G_{(134)}$.

To prove (i), note that since $M_{22}$ contains a unique class of involutions, they must remain involutions in $\bar{M}_{22}$; otherwise the Sylow 2-subgroup of $\bar{M}_{22}$ would be cyclic or generalized quaternion. Consequently elements of order 4 and 8 must also remain the same order in $\bar{M}_{22}$. Let $\bar{P}_{1} / Z_{2} \simeq P_{1}$ be a covering of $P_{1}$. The only coverings of elementary abelian 2 -subgroups in which the involutions remain involutions are again elementary abelian 2-groups. Thus $E$ is covered by $\bar{E}$, an elementary abelian group of order 32 , and $\bar{P}_{1}$ is the semi-direct product of $\bar{E}$ by $\left\{\bar{B}_{1}, \bar{B}_{2}\right\} \simeq Z_{2} \times Z_{2}$. By a suitable choice of basis in $\bar{E}$ we may take

$$
\bar{B}_{1}=\left(\frac{1 \mid \vec{c}_{1}}{0 \mid B_{1}}\right) \quad \bar{B}_{2}=\left(\frac{1 \mid \vec{c}_{2}}{0 \mid B_{2}}\right)
$$


where $\vec{c}_{i}=\left(c_{i 1}, c_{i 2}, c_{i 3}, c_{i 4}\right), i=1,2$, are as yet unspecified rows of four numbers from $G F(2)$. Use $\left(\bar{B}_{1}\right)^{2}=\left(\bar{B}_{2}\right)^{2}=\left(\bar{B}_{1} \bar{B}_{2}\right)^{2}=1$. One finds that $c_{11}=c_{12}=c_{21}=c_{23}$ $=c_{13}+c_{22}=0$. By a suitable change of basis in $\bar{E}, \bar{B}_{i} \rightarrow W \bar{B}_{i} W^{-1}$ where

$$
W=\left(\begin{array}{c|c}
1 \mid c_{13} c_{14} c_{24} 0 \\
0
\end{array}\right)
$$

we may in fact assume that $\vec{c}_{1}=\vec{c}_{2}=\overrightarrow{0}$. Therefore $\bar{P}_{1} \simeq P_{1} \times Z_{2}$. Since $G_{(1341)}$ equals its commutator subgroup, any covering of it in $\bar{M}_{22}$ must be $G_{(1344)} \times Z_{2}$.

Apply the inducing argument with $S=G_{(1344)}(\pi=1+21+55+154+99)$. The (1), $(3)^{6},(5)^{4},(8)^{2}(4)(2)$, and both $(7)^{3}$ and $(11)^{2}$ classes must split for the usual reasons. The other classes are forced to split by $(*)$. Due to the large index of $G_{(1314}$ in $M_{22}$, no useful information is obtained by restricting $\phi^{*}$ back to $G_{(1314)} \times Z_{2}$.

To prove (iii) we first note that the only central extension of $Z_{2}$ by $D_{8}$ in which involutions remain involutions is $D_{3} \times Z_{2}$. Therefore $\bar{P}$ is the semi-direct product of $\bar{E}$ by $\left\{\bar{B}_{1}, \bar{B}_{2}, \bar{B}_{3}\right\} \simeq D_{s}$, where

$$
\bar{B}_{i}=\left(\frac{1 \mid \vec{c}_{i}}{0 \mid B_{i}}\right) \quad i=1,2,3
$$

Repeating the argument used in proving (i) we may assume $\vec{c}_{1}=\vec{c}_{2}=0$. Using $\left(\bar{B}_{3}\right)^{2}=I$ and $\left(\bar{B}_{2} \bar{B}_{3}\right)^{2}=\bar{B}_{1}$ we find that $\vec{c}_{3}=(0,0,0, d)$, where $d=0$ or 1 . If $d=1$ then it is easily seen that certain classes of elements of order 4 in $P$ do not split in $\bar{P}$, as for example the class containing $\left(B_{1}, \vec{b}\right)$ with $\vec{b}=(0,0,0,1)$. The classes of $M_{22}$ containing these elements would thus not split in $\bar{M}_{22}$, contradicting the result (ii). Therefore $d=0, \bar{P} \simeq P \times Z_{2}$ and $\bar{M}_{22} \simeq M_{22} \times Z_{2}$.

The authors apologize for this round-about proof and would not be surprised if a shorter argument sufficed.

In the 3-fold covering of $M_{22}$ all but the $(3)^{6}$ class splits. There are thus 11 pairs of projective characters. The degrees are $21,45,45,99,105,105,210$, $231,231,330,384$. Table 6 gives the values of the 21-dimensional character.

In the final section we present an interesting application of some work of Brauer [7]. The method could also be used to give considerable information in the case of $M_{22}$. Unfortunately, it did not lead to a complete answer. For $M_{23}$, as will be seen, the extra prime allows a unique conclusion. 


\section{§ 5. The Block Theory Argument}

Suppose $M_{23}$ has a covering $\bar{M}_{23} / Z_{2} \simeq M_{23}$. We shall find the degrees of the irreducible characters of $\bar{M}_{23}$ which are faithful on $Z_{2}$. These projective characters will be distinguished with a prime. If $\bar{M}_{23}$ is a proper covering, their degrees must be even. We shall see that this leads to a contradiction.

$M_{23}$ has characters $896,896^{*}$. Since $896=2^{7} \cdot 7$, as characters of $\bar{M}_{23}$ they belong to 2-blocks of defect 1 . By block theory there must exist $896^{\prime}, 896^{\prime *}$. 23, 11,7 all divide $\left|\bar{M}_{23}\right|$ to the first power. The centralizer of $Z_{p}$ in $\bar{M}_{23}$ is $Z_{p} \times Z_{2}, p=23,11$. Hence there exists exactly one block $B^{\prime}(p)$ of $\bar{M}_{23}$ of defect 1 distinct from the principal block $\left.B^{(} p\right)$ for $p=23,11$. For $p=7$ there exist two blocks $B^{\prime}(7), B^{\prime \prime}(7)$ of $\bar{M}_{23}$ of defect 1 consisting of projective characters. Since $896 \equiv 5(\bmod 11)$, the characters $896^{\prime}, 896^{\prime *}$ are exceptional in $B^{\prime}(11)$ (see Brauer [7]). The remaining projective degrees are then congruent to

$$
\begin{array}{r}
0, \pm 1, \pm 11 \quad(\bmod 23) \\
0, \pm 1 \quad(\bmod 11) \\
0, \pm 1, \pm 3 \quad(\bmod 7)
\end{array}
$$

The only numbers dividing $\left|\bar{M}_{23}\right|$ and congruent $(\bmod 23)$ to $\pm 1, \pm 11$ are

$$
\begin{aligned}
1: & 1,24,70,231,576,990,1680 \\
-1: & 22,45,160,252,528,896,1540 \\
11: & 11,80,126,264,448,770,1920 \\
-11: & 12,35,288,495,840,1760,2772 .
\end{aligned}
$$

Now exclude (i) odd degrees, (ii) degrees $\neq 0, \pm 1(\bmod 11)$, (iii) degrees $\neq 0$, $\pm 1, \pm 3(\bmod 7),($ iv) 2772 , since it occurs twice if at all, and then is too large. This leaves

$$
\begin{aligned}
1: & 990 \\
-1: & 22,252,528,1540 \\
11: & 770 \\
-11: & 1760 .
\end{aligned}
$$

Since there do not exist elements of order 11.23 in $M_{23}$, we can apply the results of Stanton [8], $\S 5$ to the intersection $B^{\prime}(11) \cap B^{\prime}(23)$, and conclude that the above remaining possible projective degrees cannot occur. This contradiction can of course be traced to the exclusion of odd degrees, 


\section{REFERENCES}

[1] E. Witt, Die 5-fach transitiven Gruppen von Mathieu, Abhand. Math. Sem. Hamburg, Vol 12 (1938), pp. 256-264.

[2] I. Schur, Über die Darstellung der endlichen Gruppen durch gebrochene lineare Substitutionen, Jour. für Math. (Crelle), Vol 127 (1904), pp. 20-50; Untersuchungen über die Darstellung der endlichen Gruppen durch gebrochene lineare Substitutionen, ibid, Vol 132 (1907), pp. 85-137; Über die Darstellung der symmetrischen und der alternierenden Gruppe durch gebrochene lineare Substitutionen, ibid, Vol 139 (1911), pp. 155-250.

[3] G. Frobenius, Über die Charaktere der mehrfach transitive Gruppen, Sitz. Preuss. Akad. Wissen. (1904), pp. 558-571.

[4] D. E. Littlewood, The Theory of Group Characters, 2nd. Ed. Oxford (1950).

[5] G. A. Miller, The group of isomorphisms of the simple groups whose degrees are less than 15, Archiv der Math. und Physik, Vol 12 (1907), pp. 249-251; Transitive groups of degree $p=2 q+1, p$ and $q$ being prime numbers, Quarterly Jour. of Math. Vol 39 (1908), pp. 210-216.

[6] R. Swan, The p-period of a finite group, Ill. Jour. of Math. Vol 4 (1960), pp. 341-346.

[7] R. Brauer, On groups whose order contains a prime number to the first power I, Amer. Jour. Math. Vol 64 (1942), pp. 401-420.

[8] R. G. Stanton, The Mathieu groups, Can. Jour. Math. Vol 3 (1951), pp. 164-174.

\section{Tables}

In Tables 1, 2, 3 the first column describes the conjugacy class by giving its cycle structure in the natural permutation representation of that group. The second column is the order $h$ of the centralizer subgroup of an element in that class. A character written with a bar above it denotes a pair of complexconjugate characters. Recall that the orders of the Mathieu groups are $h_{11}=$ 11.10.9.8, $h_{12}=12 . h_{11}, h_{22}=22.21 .20 .48, h_{23}=23 . h_{22}, h_{24}=24 . h_{23}$.

In Table 4 we give the character $\pi$ (see $\S 2$ of part II) for the $M_{11}$ subgroup in $M_{12}$. We only list those classes on which the character is non-zero. In Table 5 we list the values of $\pi$ for $M_{23}$ in $M_{24}$ only on those classes for which they are needed in $\S 3$ of part II. In Table 6 we only give a single value for the projective character. The other values on each splitting class are multiples of the given value by $\frac{1}{2}(-1 \pm i \sqrt{3})$. 
TABLE 1. Characters of $M_{11}$

\begin{tabular}{lr|rrrrrrrr}
\hline$(1)$ & $h_{11}$ & 1 & 10 & 11 & 55 & 45 & 44 & $\overline{16}$ & $\overline{10}$ \\
$(2)^{4}$ & 48 & 1 & 2 & 3 & -1 & -3 & 4 & 0 & -2 \\
$(4)^{2}$ & 8 & 1 & 2 & -1 & -1 & 1 & 0 & 0 & 0 \\
$(3)^{3}$ & 18 & 1 & 1 & 2 & 1 & 0 & -1 & -2 & 1 \\
$(5)^{2}$ & 5 & 1 & 0 & 1 & 0 & 0 & -1 & 1 & 0 \\
$(8)(2)$ & 8 & 1 & 0 & -1 & 1 & -1 & 0 & 0 & $\pm i \sqrt{2}$ \\
$(8)(2)$ & 8 & 1 & 0 & -1 & 1 & -1 & 0 & 0 & $\mp i \sqrt{2}$ \\
$(6)(3)(2)$ & 6 & 1 & -1 & 0 & -1 & 0 & 1 & 0 & 1 \\
$(11)$ & 11 & 1 & -1 & 0 & 0 & 1 & 0 & $z$ & -1 \\
$(11)$ & 11 & 1 & -1 & 0 & 0 & 1 & 0 & $\bar{z}$ & -1 \\
\hline
\end{tabular}

where $z=1 / 2(-1 \pm i \sqrt{11})$.

TABLE 2. Characters of $M_{22}$

\begin{tabular}{lr|rrrrrrrrrr}
\hline \hline 1$)$ & $h_{22}$ & 1 & 21 & 55 & 154 & 210 & $\overline{280}$ & 231 & 385 & 99 & $\overline{45}$ \\
$(2)^{8}$ & 384 & 1 & 5 & 7 & 10 & 2 & -8 & 7 & 1 & 3 & -3 \\
$(3)^{6}$ & 36 & 1 & 3 & 1 & 1 & 3 & 1 & -3 & -2 & 0 & 0 \\
$(5)^{4}$ & 5 & 1 & 1 & 0 & -1 & 0 & 0 & 1 & 0 & -1 & 0 \\
$(4)^{4}(2)^{2}$ & 16 & 1 & 1 & -1 & 2 & -2 & 0 & -1 & 1 & -1 & 1 \\
$(4)^{4}(2)^{2}$ & 32 & 1 & 1 & 3 & -2 & -2 & 0 & -1 & 1 & 3 & 1 \\
$(7)^{3}$ & 7 & 1 & 0 & -1 & 0 & 0 & 0 & 0 & 0 & 1 & $z_{1}$ \\
$(7)^{3}$ & 7 & 1 & 0 & -1 & 0 & 0 & 0 & 0 & 0 & 1 & $\bar{z}_{1}$ \\
$(8)^{2}(4)(2)$ & 8 & 1 & -1 & 1 & 0 & 0 & 0 & -1 & 1 & -1 & -1 \\
$(6)^{2}(3)^{2}(2)^{2}$ & 12 & 1 & -1 & 1 & 1 & -1 & 1 & 1 & -2 & 0 & 0 \\
$(11)^{2}$ & 11 & 1 & -1 & 0 & 0 & 1 & $z_{2}$ & 0 & 0 & 0 & 1 \\
$(11)^{2}$ & 11 & 1 & -1 & 0 & 0 & 1 & $\bar{z}_{2}$ & 0 & 0 & 0 & 1 \\
\hline & where $z_{1}=1 / 2(-1 \pm i \sqrt{7})$ and $z_{2}=1 / 2(-1 \pm i \sqrt{11})$. & &
\end{tabular}


TABLE 3. Characters of $M_{23}$

\begin{tabular}{lr|rrrrrrrrrrrr}
\hline$(1)$ & $h_{23}$ & 1 & 22 & 230 & 231 & $\overline{770}$ & 1035 & 2024 & $\overline{45}$ & $\overline{990}$ & 231 & 253 & $\overline{896}$ \\
$(2)^{8}$ & 2688 & 1 & 6 & 22 & 7 & -14 & 27 & 8 & -3 & -18 & 7 & 13 & 0 \\
$(3)^{6}$ & 180 & 1 & 4 & 5 & 6 & 5 & 0 & -1 & 0 & 0 & -3 & 1 & -4 \\
$(5)^{4}$ & 15 & 1 & 2 & 0 & 1 & 0 & 0 & -1 & 0 & 0 & 1 & -2 & 1 \\
$(4)^{4}(2)^{2}$ & 32 & 1 & 2 & 2 & -1 & -2 & -1 & 0 & 1 & 2 & -1 & 1 & 0 \\
$(7)^{3}$ & 14 & 1 & 1 & -1 & 0 & 0 & -1 & 1 & $z_{1}$ & $z_{1}$ & 0 & 1 & 0 \\
$(7)^{3}$ & 14 & 1 & 1 & -1 & 0 & 0 & -1 & 1 & $\bar{z}_{1}$ & $\bar{z}_{1}$ & 0 & 1 & 0 \\
$(8)^{2}(4)(2)$ & 8 & 1 & 0 & 0 & -1 & 0 & 1 & 0 & -1 & 0 & -1 & -1 & 0 \\
$(6)^{2}(3)^{2}(2)^{2}$ & 12 & 1 & 0 & 1 & -2 & 1 & 0 & -1 & 0 & 0 & 1 & 1 & 0 \\
$(11)^{2}$ & 11 & 1 & 0 & -1 & 0 & 0 & 1 & 0 & 1 & 0 & 0 & 0 & $z_{2}$ \\
$(11)^{2}$ & 11 & 1 & 0 & -1 & 0 & 0 & 1 & 0 & 1 & 0 & 0 & 0 & $\bar{z}_{2}$ \\
$(15)(5)(3)$ & 15 & 1 & -1 & 0 & 1 & 0 & 0 & -1 & 0 & 0 & $z_{3}$ & 1 & 1 \\
$(15)(5)(3)$ & 15 & 1 & -1 & 0 & 1 & 0 & 0 & -1 & 0 & 0 & $\bar{z}_{3}$ & 1 & 1 \\
$(14)(7)(2)$ & 14 & 1 & -1 & 1 & 0 & 0 & -1 & 1 & $-z_{1}$ & $z_{1}$ & 0 & -1 & 0 \\
$(14)(7)(2)$ & 14 & 1 & -1 & 1 & 0 & 0 & -1 & 1 & $-\bar{z}_{1}$ & $\bar{z}_{1}$ & 0 & -1 & 0 \\
$(23)$ & 23 & 1 & -1 & 0 & 1 & $z_{4}$ & 0 & 0 & -1 & 1 & 1 & 0 & -1 \\
$(23)$ & 23 & 1 & -1 & 0 & 1 & $\bar{z}_{4}$ & 0 & 0 & -1 & 1 & 1 & 0 & -1 \\
\hline
\end{tabular}

where $z_{1}=1 / 2(-1 \pm i \sqrt{7}), z_{2}=1 / 2(-1 \pm i \sqrt{11}), z_{3}=1 / 2(-1 \pm i \sqrt{15})$, and $z_{4}=1 / 2(-1 \pm i \sqrt{ } \overline{23})$.

TABLE 4. Character of $M_{11}$ in $M_{12}$

\begin{tabular}{c|rrrrrrrrr}
\hline class & $(1)$ & $(2)^{4}$ & $(4)^{2}$ & $(3)^{3}$ & $(5)^{2}$ & $(8)(2)$ & $(6)(3)(2)$ & $(11)$ & $(11)$ \\
\hline$h$ & $h_{12}$ & 192 & 32 & 54 & 10 & 8 & 6 & 11 & 11 \\
\hline$\pi$ & 12 & 4 & 4 & 3 & 2 & 2 & 1 & 1 & 1 \\
\hline
\end{tabular}

TABLE 5. Part of the Character of $M_{23}$ in $M_{24}$

\begin{tabular}{c|cccccc}
\hline class & $(2)^{8}$ & $(4)^{4}(2)^{2}$ & $(8)^{2}(4)(2)$ & $(6)^{2}(3)^{2}(2)^{2}$ & $(14)(7)(2)+$ & $(14)(7)(2)-$ \\
\hline$h$ & $21.2^{10}$ & 128 & 16 & 24 & 14 & 14 \\
\hline$\pi$ & 8 & 4 & 2 & 2 & 1 & 1 \\
\hline
\end{tabular}

TABLE 6. Projective Character of $M_{22}$

\begin{tabular}{|c|c|c|c|c|c|c|c|c|c|c|c|c|}
\hline class & (1) & $(2)^{8}$ & $(3)^{6}$ & $(5)^{4}$ & $(4)^{4}(2)^{2}$ & $(4)^{4}(2)^{2}$ & $(6)^{2}(3)^{2}(2)^{2}$ & $(7)^{3}$ & $(7)^{3}$ & $(8)^{2}(4)(2)$ & $(11)^{2}$ & $(11)^{2}$ \\
\hline$\chi$ & 21 & 5 & 0 & 1 & 1 & 1 & 2 & 0 & 0 & -1 & -1 & -1 \\
\hline
\end{tabular}

Department of Mathematics

University of California

Berkeley, California

University of Illinois

Chicago, Illinois 